\title{
The Nucleus Retroambiguus Control of Respiration
}

\author{
Hari H. Subramanian and Gert Holstege \\ Center for Uroneurology, University of Groningen, University Medical Center Groningen, 9700 RB Groningen, The Netherlands
}

The role of the nucleus retroambiguus (NRA) in the context of respiration control has been subject of debate for considerable time. To solve this problem, we chemically (using D, L-homocysteic acid) stimulated the NRA in unanesthetized precollicularly decerebrated cats and studied the respiratory effect via simultaneous measurement of tracheal pressure and electromyograms of diaphragm, internal intercostal (IIC), cricothyroid (CT), and external oblique abdominal (EO) muscles. NRA-stimulation $0-1 \mathrm{~mm}$ caudal to the obex resulted in recruitment of IIC muscle and reduction in respiratory frequency. NRA-stimulation 1-3 mm caudal to the obex produced vocalization along with CT activation and slight increase in tracheal pressure, but no change in respiratory frequency. NRA-stimulation 3-5 mm caudal to the obex produced CT muscle activation and an increase in respiratory frequency, but no vocalization. NRA-stimulation 5-8 $\mathrm{mm}$ caudal to the obex produced EO muscle activation and reduction in respiratory frequency. A change to the inspiratory effort was never observed, regardless of which NRA part was stimulated. The results demonstrate that NRA does not control eupneic inspiration but consists of topographically separate groups of premotor interneurons each producing detailed motor actions. These motor activities have in common that they require changes to eupneic breathing. Different combination of activation of these premotor neurons determines the final outcome, e.g., vocalization, vomiting, coughing, sneezing, mating posture, or child delivery. Higher brainstem regions such as the midbrain periaqueductal gray (PAG) decides which combination of NRA neurons are excited. In simple terms, the NRA is the piano, the PAG one of the piano players.

\section{Introduction}

The nucleus retroambiguus (NRA) in the cat is a column of neurons in the ventrolateral medulla extending from 1 to $8 \mathrm{~mm}$ caudal to the obex (Olszewski and Baxter, 1954; Berman, 1968). The NRA receives afferents from several respiration-related nuclei such as the Bötzinger and pre-Bötzinger complex, the periambigual region, the ventrolateral part of the medulla caudal to the facial nucleus, the ventrolateral parabrachial nucleus, the nucleus Kölliker-Fuse and the retrotrapezoid nucleus in the pons (Gerrits and Holstege, 1996). Afferents also arise from the solitary nucleus and two cell groups in the ventral part of the medullary medial tegmental field, one at the level of facial nucleus and one just rostral to hypoglossal nucleus (Gerrits and Holstege, 1996). Outside the caudal brainstem, the NRA receives strong projections from the lateral, ventrolateral, and to a lesser extent the dorsomedial part of the caudal midbrain periaqueductal gray (PAG) (Holstege, 1989), but not from any other suprapontine brain structure (Holstege, 1991). The NRA projects directly to the laryngeal and pharyngeal motoneurons in the nucleus ambiguus in the lateral medulla (Holstege, 1989; Boers et al., 2002) and to the motoneurons of the diaphragm, external and internal intercostal, abdominal (Merril, 1970, 1974; Holstege and Kuypers,

Received Feb. 5, 2009; accepted Feb. 20, 2009

H.H.S. was a recipient of an Australian National Health and Medical Research Council Neuroscience scholarship for postgraduate research, Balmain Hospital New South Wales research assistantship, and support grants from the Faculty of Health Sciences, The University of Sydney. We thank Dr. Ron Balnave (School of Medical Sciences) and the Gordon-Craig Animal Experimental Laboratory at The University of Sydney.

Correspondence should be addressed to Dr. Gert Holstege at the above address. E-mail: G.Holstege@med.umcg.nl.

D01:10.1523/JNEUROSCI.0607-09.2009

Copyright $\odot 2009$ Society for Neuroscience $\quad$ 0270-6474/09/293824-09\$15.00/0
1982), pelvic floor, upper leg, and hip muscles (Holstege and Tan, 1987; Vanderhorst and Holstege, 1995) in the spinal cord. The NRA is also called caudal component of the ventral respiratory group (VRG) (Long and Duffin, 1986) because its rostral part contains two types of inspiration-related neurons while its caudal part contains expiration-related neurons (Merril, 1970, 1974; Long and Duffin, 1986).

The question is whether the NRA really contributes to the eupneic (normal, quiet breathing is called eupnea) inspiratory drive as proposed by Merril $(1970,1974)$ or whether it only contains premotor interneurons of motoneurons innervating respiration-related muscles. To solve this problem, in unanesthetized precollicularly decerebrated cats, we used excitatory amino acid microinjections to stereotaxically stimulate different portions of the NRA and (in one case) the midbrain periaqueductal gray, and simultaneously studied the changes in tracheal pressure and in the electromyograms of diaphragm, cricothyroid, and intercostal and abdominal muscles.

\section{Materials and Methods}

Surgery. Approval for these studies was obtained from The University of Sydney institutional Animal Care Ethics Committee. Cats ( $n=8$, weighing between 2.2 and $4.5 \mathrm{~kg}$ ) were anesthetized in a box filled with a mixture of isoflurane and oxygen delivered by a ventilatory pump. After the induction, the anesthesia was maintained through a face-mask, while femoral arterial and venous catheters were inserted. Using the venous catheter, sodium thiopental $(10-20 \mathrm{mg} / \mathrm{kg})$ was injected to enable the insertion of an endotracheal catheter, which was then used for subsequent continued administration of isoflurane. The cat's head was then secured in a stereotaxic frame with the body suspended from the frame by straps. Regardless of the type, all anesthetics suppress breathing. Since this depressing effect is not present in decerebrate preparations, the cats were decerebrated at the precollicular level. To allow access to the mid- 

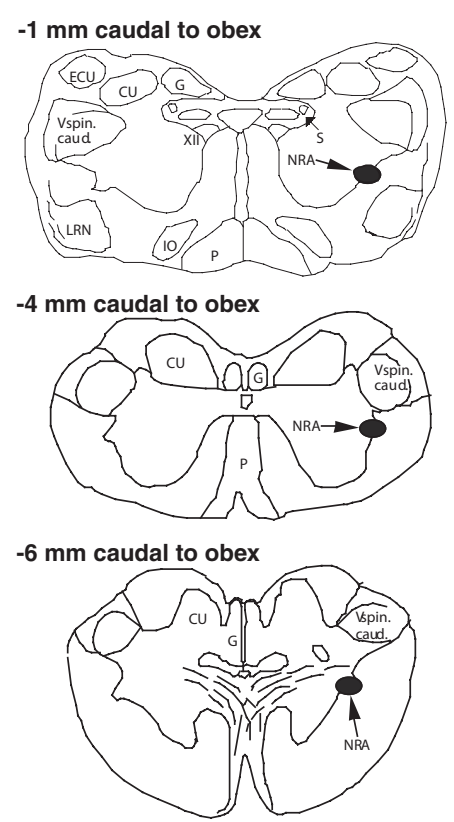

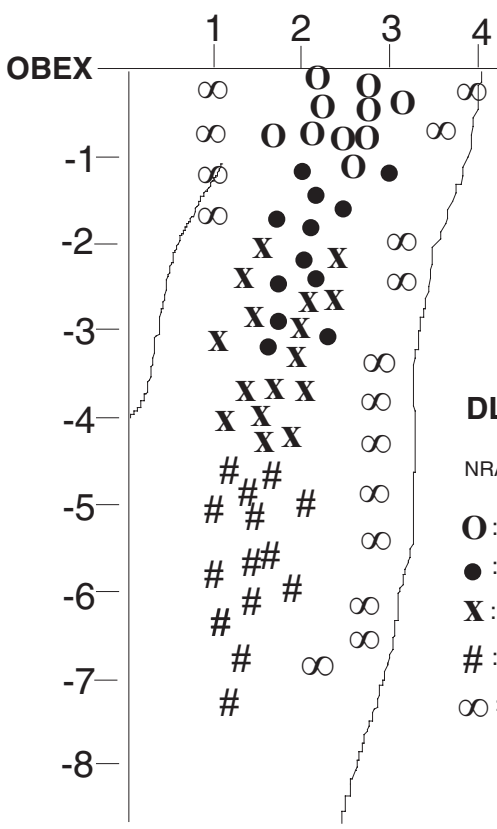

\section{DLH INJECTION SITES}

NRA rostrocaudal extension according to the atlas of Olzewski and Baxter (1954).

$\mathbf{O}$ :Internal Intercostal muscle excitation

- :Cricothyroid muscle excitation and tracheal pressure increase

$\mathbf{X}$ :Cricothyroid muscle excitation only

\# :External oblique abdominal muscle excitation

$\infty$ : No effect on tracheal pressure, IIC/CT/EO muscles and no vocalization

Figure 1. The NRA stimulation sites and responses evoked. The zero reference was the midline. The location of the NRA in terms of millimeter lateral to the midline was quite different in the eight cats. In the same cat, we never had any effect $2 \mathrm{~mm}$ apart from a positive injection site in laterality. CU, Cuneate nucleus; ECU, external cuneate nucleus; $G$, gracile nucleus; I0, inferior olive; LRN, lateral reticular nucleus; $\mathrm{P}$, pyramidal tract; $\mathrm{S}$, solitary complex; $\mathrm{V}$ spin. caud, spinal trigeminal complex pars caudalis; XII, hypoglossal nucleus.

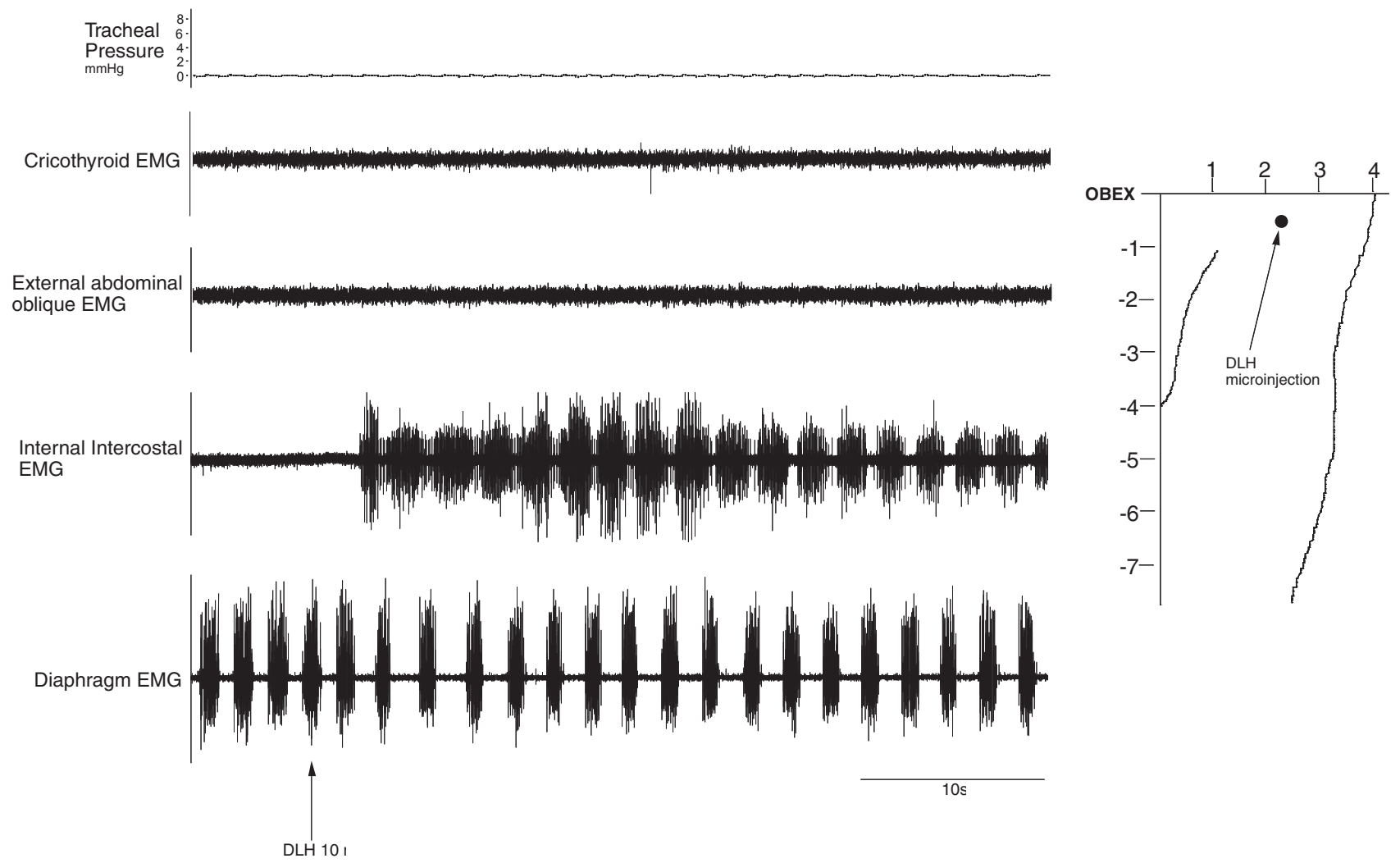

Figure 2. Inspiratory shortening and expiratory lengthening induced from the NRA $0-1 \mathrm{~mm}$ caudal to the obex. Note the recruitment of internal intercostal expiratory muscles.

brain, two burr holes were drilled in the skull on either side of the sagittal sinus. An occipital craniotomy was performed to allow access to the caudal brainstem. The dura was then incised and the medial part of the cortex was suctioned. After ligation and removal of a portion of the sagittal sinus, precollicular decerebration was performed using suction diathermia, a surgical technique that uses pulsations of electrical energy to generate heat and cauterize blood vessels to prevent excessive bleeding while suctioning brain tissue. Bleeding during decerebration was reduced by lowering the mean arterial pressure from $100-130$ $\mathrm{mmHg}$ to $65-70 \mathrm{mmHg}$ by raising the level of anesthesia. All brain tissue rostral to the superior colliculus including the entire diencephalon was removed. After completion of decerebration, anesthesia was discontin- 


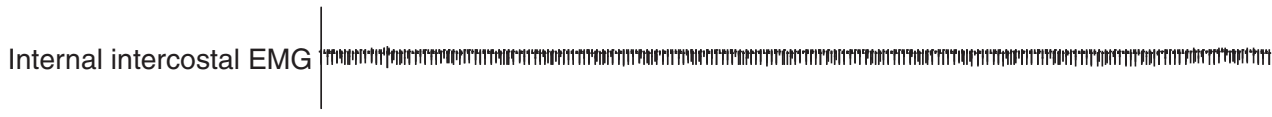

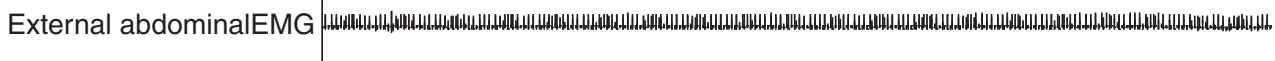

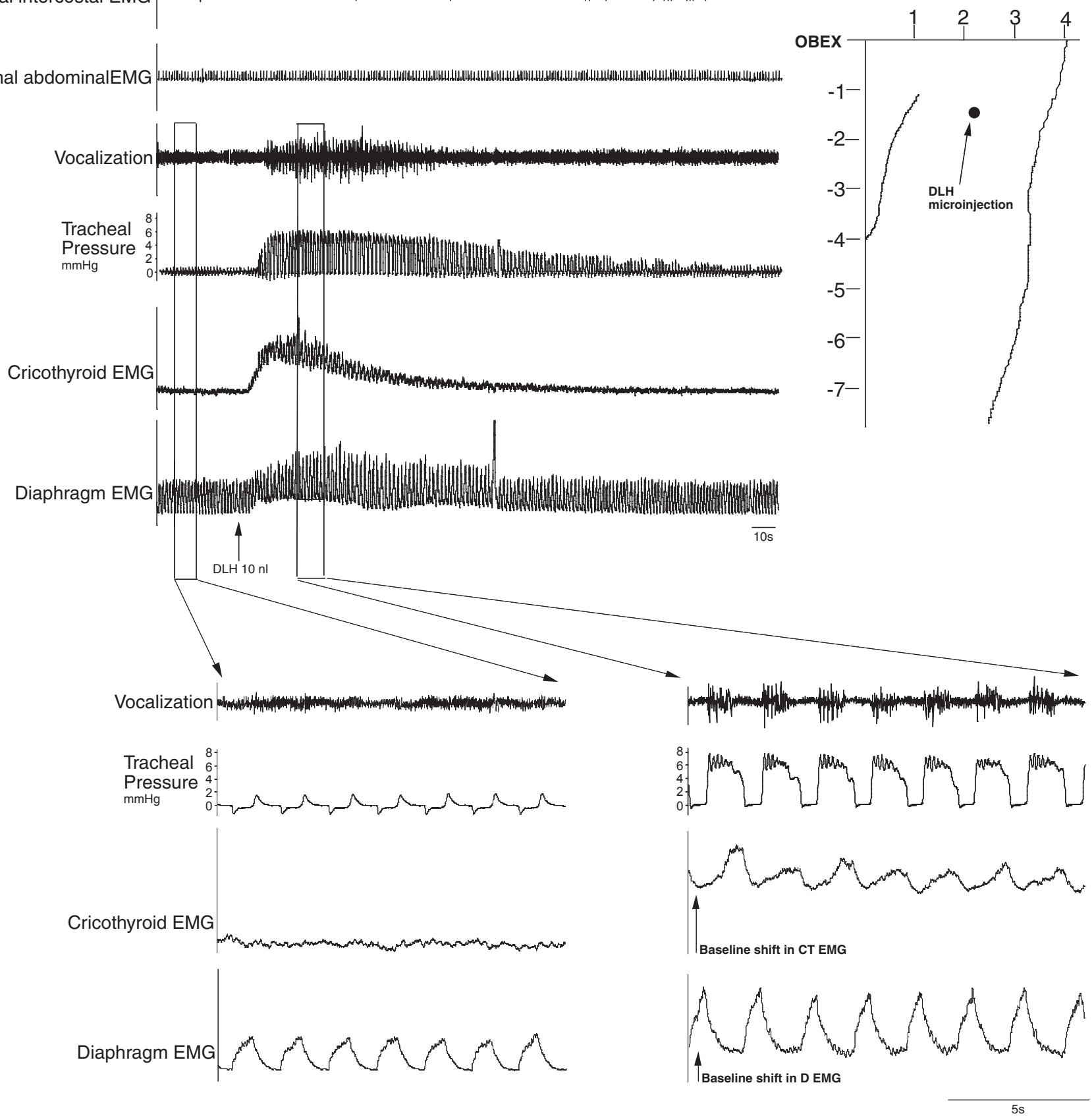

Figure 3. Vocalizations induced from the NRA 1-3 mm caudal to the obex. Note the baseline shift in the EMG of cricothyroid and diaphragm muscles during vocalization. Integrated EMG time constants, $100 \mathrm{~ms}$.

ued. The cat started to breath spontaneously and the mean arterial pressure returned to $100-130 \mathrm{mmHg}$ within $30-60 \mathrm{~min}$. A thermostatic infrared lamp was used to maintain the animal body temperature at $37.5-38.5^{\circ} \mathrm{C}$. The end tidal $\mathrm{CO}_{2}$ (measured by means of a Morgan 901 gas analyzer) was intermittently monitored. Fluid supplements were administered via the femoral intravenous catheter.

Chemical stimulation of the NRA. Excitatory amino acid [D, L-homocysteic acid (DLH), $200 \mathrm{~mm}$, Sigma] microinjections were used for chemical stimulation of the NRA. For microinjections of DLH, a single barrel glass-micropipette (tip diameter, 10-30 $\mu \mathrm{m}$ ) was inserted into the NRA guided by stereotaxic coordinates. A pressure system (Picospritzer II, Parker Instrumentation) was used to deliver the microinjections. The volume injected was determined using a precalibrated scale. An interval of $25 \mathrm{~min}$ between microinjections was provided to eliminate any residual effects of the previous injection. Rhodamine beads were added to the DLH solution for later histological identification of the injection sites. At the end of each experiment, the animal was deeply anesthetized and perfused transcardially with $0.9 \%$ saline followed by $4 \%$ paraformaldehyde in phosphate buffer $(\mathrm{pH}=7.2)$. After perfusion, the brain was removed and stored in $4 \%$ formaldehyde for $2 \mathrm{~h}$. It was then transferred to a $30 \%$ sucrose/formaldehyde mixture for at least $48 \mathrm{~h}$ to prevent ice crystal formation. The caudal brainstem was cut on a freezing microtome into $50 \mu \mathrm{m}$ coronal sections. The injection sites marked with rhodamine were identified using fluorescent microscopy and were represented on standard drawings according to Berman's (1968) cat stereotaxic atlas.

EMG recording. Unilateral EMG activity was recorded using bipolar Teflon-coated, multistranded stainless steel electrodes, bared for $2 \mathrm{~mm}$ at 

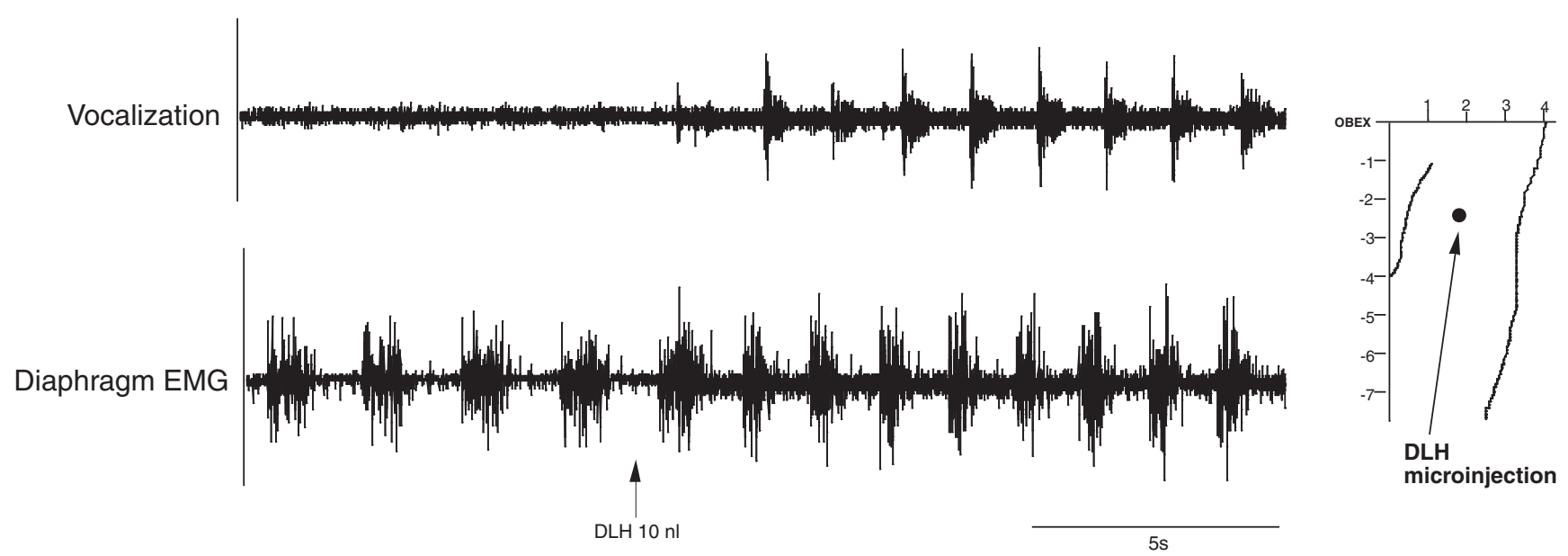

Figure 4. Comparison of diaphragm EMG before and after NRA-induced vocalization. No qualitative change to the diaphragm signal during vocal effect is evident, only a small increase in the amplitude.

their tips. The electrodes were surgically implanted in the crural diaphragm, cricothyroid, internal intercostal and external abdominal oblique muscles. Tracheal pressure (TP) was recorded through a 19 gauge needle inserted into the trachea and connected to a differential pressure transducer. Mean arterial pressure was recorded through the catheter placed in the femoral artery and obtained from the low pass filtered pressure pulses. The mouth of the cat was kept open and vocalization was recorded with a microphone placed $10 \mathrm{~cm}$ from the mouth.

Data analysis. The bandpass filtered $(0.1-5 \mathrm{KHz})$ EMG activity of diaphragm, cricothyroid, internal intercostal, and external oblique abdominal muscles, as well as tracheal pressure, mean arterial pressure, and voice, were recorded on the "Pulse Code Modulator" (PCM); (A.R Vetter) and played back for analyses using the PCM/MacLab/Apple (Macintosh) system. Chart software (AD Instruments) running on the Apple computer was used for playing and recording of the signals for analysis. The chart software was also used for selection of the sampling rate. The recording started at least $60 \mathrm{~s}$ before the administration of DLH. After the injection, the recording continued for at least $5 \mathrm{~min}$. All the muscle activities were rectified and averaged within and across animals. Statview software running on an Apple computer was used for computation of inspiratory $(\mathrm{Ti})$ and expiratory $(\mathrm{Te})$ durations and respiratory frequency (RF), as well as for statistical comparisons, which were performed using ANOVA. The raw diaphragm EMG signal was used for measurement of $\mathrm{Ti}$ and Te. This method is extensively described in our previous study (Subramanian et al., 2008). Significant differences between the mean values were detected using Scheffe least difference test and a probability of $p<0.05$ was considered significant. Chart records have been transformed into MacDraw Pro software for data representation.

\section{Results}

Spontaneously breathing vagi-intact precollicularly decerebrated cats $(n=8)$ had the following respiratory and cardiovascular parameters (control values expressed as mean $\pm \mathrm{SE}$ ): Ti, $0.60 \pm$ $0.05 \mathrm{~s}$; Te, $0.90 \pm 0.05 \mathrm{~s}$; RF, $42 \pm 3$ breaths $/ \mathrm{min}$; blood pressure (BP), $105 \pm 5 \mathrm{mmHg}$; heart rate (HR), $199 \pm 5$ beats/min, arterial blood $\mathrm{pH}, 7.39 \pm 0.05 ; \mathrm{p}_{\mathrm{a}} \mathrm{CO}_{2}, 25.2 \pm 5.0$ Torr; $\mathrm{p}_{\mathrm{a}} \mathrm{O}_{2}, 110.5 \pm$ 10.0 Torr. During eupnea all animals had postinspiratory activity in the crural diaphragm, which lasted for $10.5 \pm 5.0 \%$ of the eupneic expiratory duration. The postinspiratory pause was between $0.01-0.04 \mathrm{~s}$. In all cases, stimulation of the NRA along its rostrocaudal axis abolished all the postinspiratory activity in the crural diaphragm. Figure 1 illustrates the location of the injection sites caudal to the obex and the elicited effects. An average of 12 with a maximum of 20 microinjections was made in each animal. At each stereotaxic position, the injections were repeated three times (with an interval of $25 \mathrm{~min}$ between the injections) for testing the reproducibility of the effect evoked from that region. The injections in the same animal in the same NRA region always had identical effects.

\section{NRA stimulation 0-1 mm caudal to the obex}

DLH injections (200 mM, $10 \mathrm{nl} ; n=10)$ in the NRA $0-1 \mathrm{~mm}$ caudal to the obex resulted in a reduction of the respiratory frequency from $42 \pm 3$ breaths/min to $30 \pm 2$ breaths/min $(p<$ $0.05)$ reflected by an increase in Te, from $0.90 \pm 0.05 \mathrm{~s}$ to $1.40 \pm$ $0.05 \mathrm{~s}(p<0.05)$. The Ti remained unchanged $(0.55 \pm 0.05 \mathrm{~s}$; $p<0.05)$ and there was no change in the diaphragm EMG amplitude, which means that the inspiratory drive remained unaltered. During this effect the internal intercostal (expiratory) muscles were recruited (Fig. 2), but the cricothyroid and abdominal muscles were not and there was no change in the tracheal pressure.

\section{NRA stimulation 1-3 mm caudal to the obex}

Microinjections (DLH, $200 \mathrm{~mm}, 10 \mathrm{nl} ; n=12$ ) in the NRA $1-3$ $\mathrm{mm}$ caudal to the obex produced vocalization, which lasted between 20 and 90 s. This vocalization cannot be described as mews, howls or hisses, as is the case with the DLH stimulation in the PAG (Subramanian et al., 2008) but a totally different subdued guttural sound. During this typical vocalization (Fig. 3), a tonic activation of the cricothyroid muscle was found without recruitment of internal intercostal or abdominal muscles. There was a slight increase of $4-6 \mathrm{~cm} \mathrm{H}_{2} \mathrm{O}$ in the tracheal pressure and also a small increase in the diaphragm EMG amplitude (Fig. 4). The NRA-induced vocalization was characterized by shortening of inspiration (Ti) from $0.60 \pm 0.05$ to $0.40 \pm 0.05 s(p<0.05)$ and lengthening of expiration (Te) from $0.90 \pm 0.05 \mathrm{~s}$ to $1.15 \pm 0.05 \mathrm{~s}$ $(p<0.05)$, leading to a virtually unchanged respiratory frequency $(40 \pm 2$ breaths/min; $p<0.05)$. The vocalization effect was the result of a combination of cricothyroid activation and the slight increase in tracheal pressure. To compare the vocalization effects elicited by stimulating the PAG and stimulating the NRA, in three cats we stimulated both the PAG and NRA $2.5 \mathrm{~mm}$ caudal to the obex. A $30 \mathrm{nl}$ DLH injection into the lateral PAG produced a large increase in tracheal pressure up to $40 \mathrm{~cm} \mathrm{H}_{2} \mathrm{O}$, while an identical $30 \mathrm{nl}$ DLH injection into the NRA in the same animal produced a small tracheal pressure increase of only $6 \mathrm{~cm} \mathrm{H}_{2} \mathrm{O}$ (Fig. 5). 
NRA stimulation 3-5 mm caudal to the obex

Stimulation (DLH, $200 \mathrm{~mm}, 10 \mathrm{nl} ; n=16$ ) of the NRA 3-5 mm caudal to the obex elicited a tonic recruitment of the cricothyroid muscle (Fig. 6). There was no vocalization, because there was no increase in tracheal pressure. During this laryngeal excitation, the respiratory frequency increased from $42 \pm 3$ breaths/min to $54 \pm 2$ breaths/min $(p<$ $0.05)$ because there was a decrease in both inspiratory duration ( $\mathrm{Ti}$ ) from $0.60 \pm 0.05 \mathrm{~s}$ to $0.30 \pm 0.05 \mathrm{~s}(p<0.05)$ and expiratory duration $(\mathrm{Te})$ from $0.90 \pm 0.05 \mathrm{~s}$ to $0.80 \pm$ $0.05 \mathrm{~s}(p<0.05)$. The internal intercostal and abdominal muscles were not recruited. There was a slight increase in the diaphragm EMG amplitude.

\section{NRA stimulation 5-8 mm caudal to the obex}

Microinjections (DLH, $200 \mathrm{~mm}, 10 \mathrm{nl} ; n=$ 14) within the NRA 5-8 mm caudal to the obex produced a tonic excitation of external abdominal oblique muscles (Fig. 7). The respiratory frequency decreased from $42 \pm 3$ breaths/min to $22 \pm 3$ breaths/min $(p<0.05)$ reflected by an increase of both the inspiratory and expiratory duration. They increased from $0.60 \pm 0.5 \mathrm{~s}$ to $0.70 \pm 0.5 \mathrm{~s}(p<0.05)$ and $0.90 \pm$ $0.5 \mathrm{~s}$ to $2.0 \pm 0.5 \mathrm{~s}(p<0.05)$, respectively. The internal intercostal and cricothyroid muscles were not recruited and no change in tracheal pressure was observed. There was a slight increase in the diaphragm EMG amplitude.

An overview of the changes to respiratory frequency and inspiratory and expiratory durations after stimulation at the various rostrocaudal levels of the NRA is shown in Figure 8.

\section{NRA does not induce inspiratory effort}

To examine the inspiratory effort produced by stimulation of the NRA at different rostrocaudal levels, the amplitude density function of the crural diaphragm EMGs was analyzed. These amplitude spectra were computed on $0.5 \mathrm{~s}$ time windows positioned at $0.7 \mathrm{~s}$ before the beginning of inspiration. For each signal, the amplitude spectra were computed for each of 10 breaths and averaged. The amplitude density spectra were normalized to the largest individual breath within the 10 breaths of control and 10 breaths of maximum effect after DLH injections in the NRA. The major peak in the amplitude density spectra occurred within the range of $200-600 \mathrm{~Hz}$ not only during eupneic inspiration, but also during the respective respiratory effects elicited in the NRA at different rostrocaudal levels (Fig. 9). No centroid frequency shift (indicator of diaphragmatic fatigue) was seen during any NRA-induced respiratory effect implying that the inspiratory effort was the same as in eupnea.

\section{Discussion}

According to Merril (1970, 1974), the NRA contains three types of inspiration-related cells, the early burst, the late-peak, and the postinspiration cells. The late-peak cells constitute the predominant population. These inspiration-related neurons project to both brainstem and spinal cord (Rikard-Bell et al., 1984, 1985). The early burst neurons send fibers to the cervical spinal cord (C3-C5) and are thought to provide premotor inspiratory drive to the phrenic motoneurons (Merril, 1970, 1974; Long and Duffin, 1986). However, in the present study, DLH stimulation of the NRA at $0-1 \mathrm{~mm}$ caudal to the obex did not significantly alter inspiration, and did not produce ventilatory responses such as augmented breaths, sustained inspiration or any other change to inspiratory effort. These results show that the rostral NRA inspiration-related neurons do not contribute to eupneic inspiratory drive. Actually, the reverse is true; stimulation of the NRA $0-1 \mathrm{~mm}$ caudal to obex resulted in an increase in the duration of expiration. This increase is brought about by the recruitment of expiration-related internal intercostal muscles, i.e., NRA cells at this level convert passive breathing into active breathing. In all likelihood, this conversion is mediated by direct NRA projections to the motoneurons of the internal intercostal muscles (Holstege and Kuypers, 1982; Rikard-Bell et al., 1984, 1985; de Troyer et al., 2005; Saywell et al., 2007). In conclusion, the NRA is not involved in eupnea, because eupnea is basically the generation and maintenance of inspiratory rhythm during which expiration is passive, using only the elastic recoil of the lungs.

\section{Respiratory function during NRA-induced vocalization}

The respiratory function during NRA-induced vocalization has never been studied before. Holstege (1989) provided anatomical evidence that neurons in the lateral and ventrolateral and to a lesser extent dorsomedial PAG have direct access to the NRA, and that the NRA neurons, in turn, project not only to the intercostal and abdominal motoneurons in the spinal cord, but also to the laryngeal and pharyngeal motoneurons in the lateral medulla. These findings led to a concept according to which the PAGNRA motoneuronal pathway serves as the "final common pathway" for vocalization. The transection experiments of Zhang et al. (1995) corroborated this concept. They examined the laryngeal and perioral component of this pathway. Our present study demonstrates that microinjections into the NRA elicit subdued guttural vocalization, but only at the level 1-3 mm caudal to the obex. The DLH dosage did not have any effect on the nature of vocalization, level of increase in tracheal pressure, or the pattern 


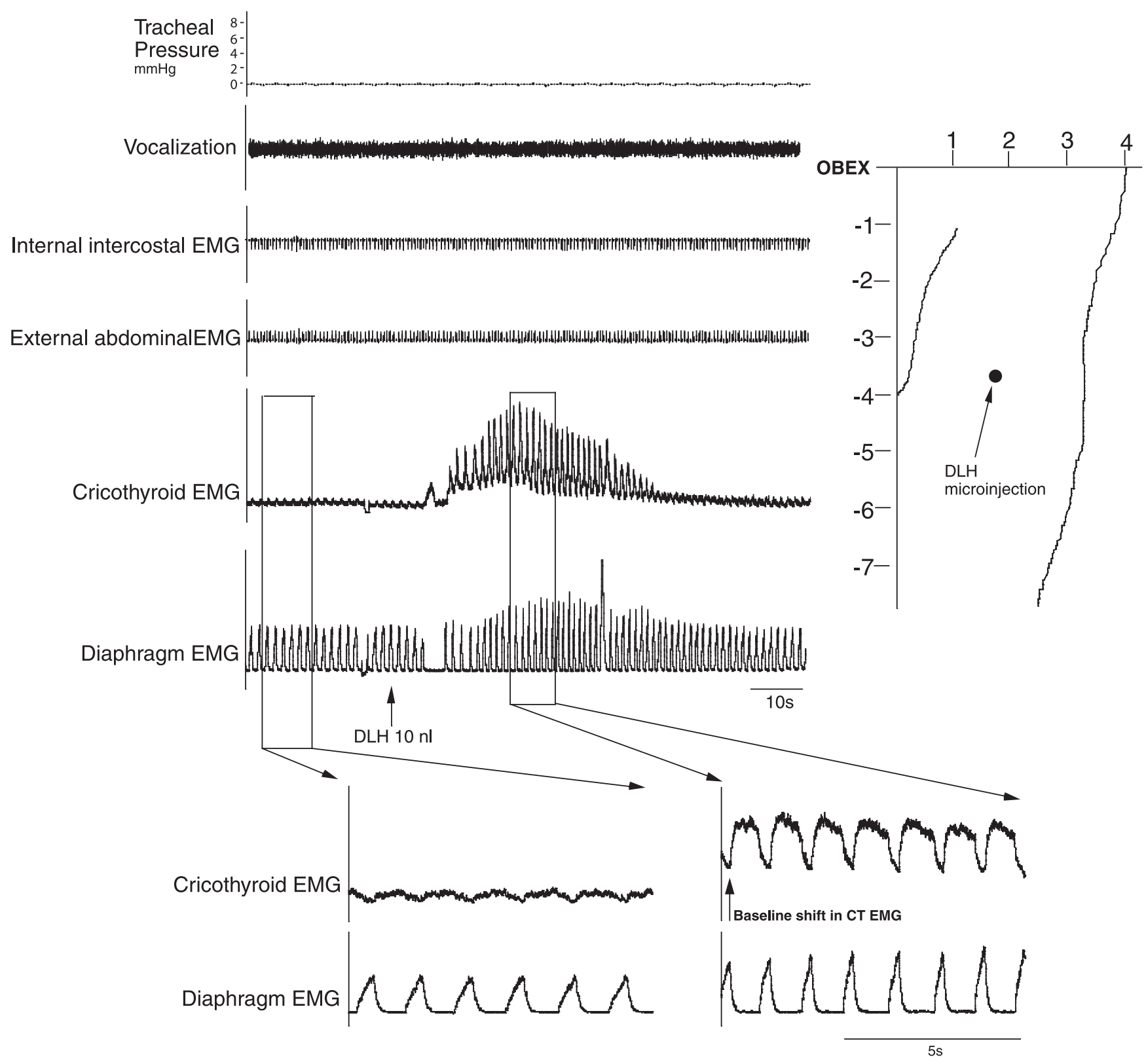

Figure 6. Recruitment of cricothyroid muscle after DLH microinjections into the NRA $3-5 \mathrm{~mm}$ caudal to the obex. Note the tonic activity of the cricothyroid muscle. Integrated EMG time constants, $100 \mathrm{~ms}$.

of EMG activity. In general, small doses of DLH injections (10-15 $\mathrm{nl}$ ) were sufficient to evoke vocalizations from the NRA, which is in strong contrast to what has been observed with DLH injections in the PAG (Subramanian et al., 2008).

Another crucial finding is that the respiratory rhythm did not undergo any change during NRA-induced vocalization. The diaphragm EMG preceding vocalization showed a small increase in its amplitude, but this increase did not have a substantial effect on the inspiratory effort. The fact that the NRA $0-5 \mathrm{~mm}$ caudal to the obex did not produce abdominal and internal intercostal activity explains why the generation of the large tracheal pressures required for vocalizations such as mews and howls could not be generated by stimulation of the NRA at these levels.

Stimulation of the NRA 3-5 mm caudal to the obex resulted in cricothyroid muscle activation (laryngeal adduction) but no vocalization, and an increase in the respiratory frequency. This in- crease seems to be a secondary effect, as it is caused by a decrease in both inspiratory and expiratory duration, but without changes in the diaphragm EMG, inspiratory effort, or activation of expiratory muscles.

NRA $5-8 \mathrm{~mm}$ caudal to the obex induces intraabdominal pressure increase The NRA $5-8 \mathrm{~mm}$ caudal to the obex is known to contain predominantly expiration-related neurons (Merril, 1970, 1974; Miller et al., 1985, 1987; Long and Duffin, 1986; Saywell et al., 2007). NRA neurons at this level have axonal projections to the motoneurons in the thoracic, lumbar, and upper sacral spinal cord and are thought to mediate the excitatory expiratory drive to the external intercostal and abdominal muscles. However, NRA cells at this level have also been shown to project to the hindlimb (Vanderhorst and Holstege, 1995) and pelvic floor muscles (Holstege and Kuypers, 1982; Holstege and Tan, 1987; Van der Horst and Holstege, 1998; Boers et al., 2006). Ac- 

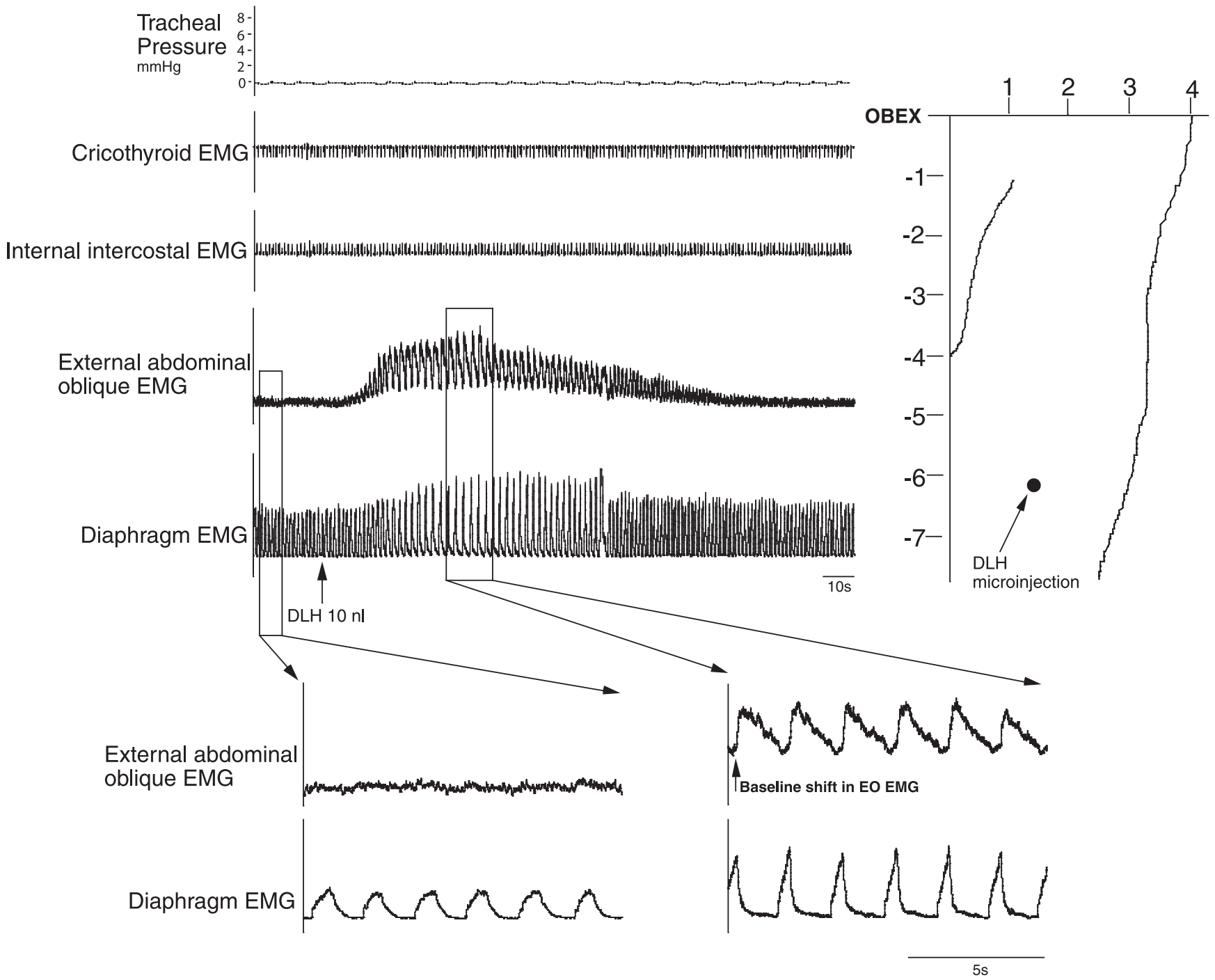

Figure 7. Recruitment of external oblique muscle after DLH microinjections into the NRA5-8mm caudal to the obex. Note the tonic activity of the external oblique abdominal muscles. Integrated EMG time constants, $100 \mathrm{~ms}$.

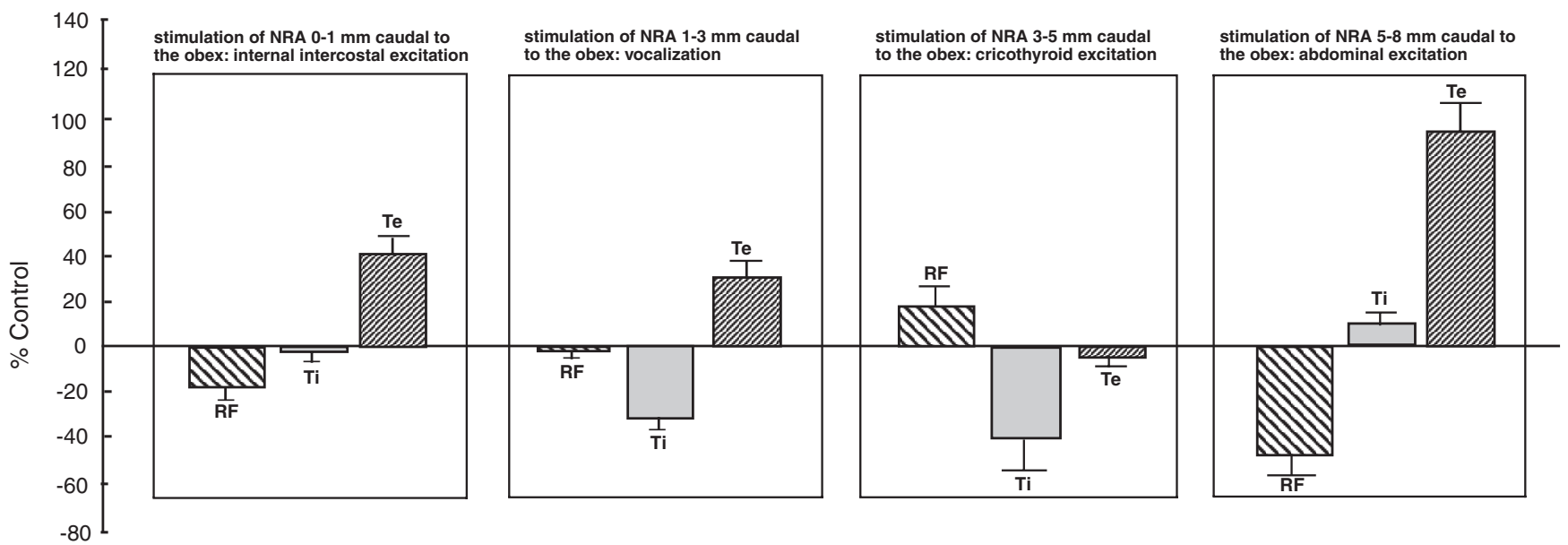

Figure 8. Histogram illustrating changes to respiratory function after stimulation of the NRA.

cording to Miller et al. (1995), the NRA also controls pudendal motoneurons innervating the pelvic floor. Ford and Kirkwood (2006) showed that the expiration-related premotor neurons in the caudal NRA do not control the hindlimb muscles, concluding that the cells groups in the NRA are functionally heterogeneous. Our study demonstrates that DLH stimulation of the NRA 5-8 $\mathrm{mm}$ caudal to the obex leads to activation of abdominal muscles resulting in an increase in expiratory duration of $>120 \%$, accom- 


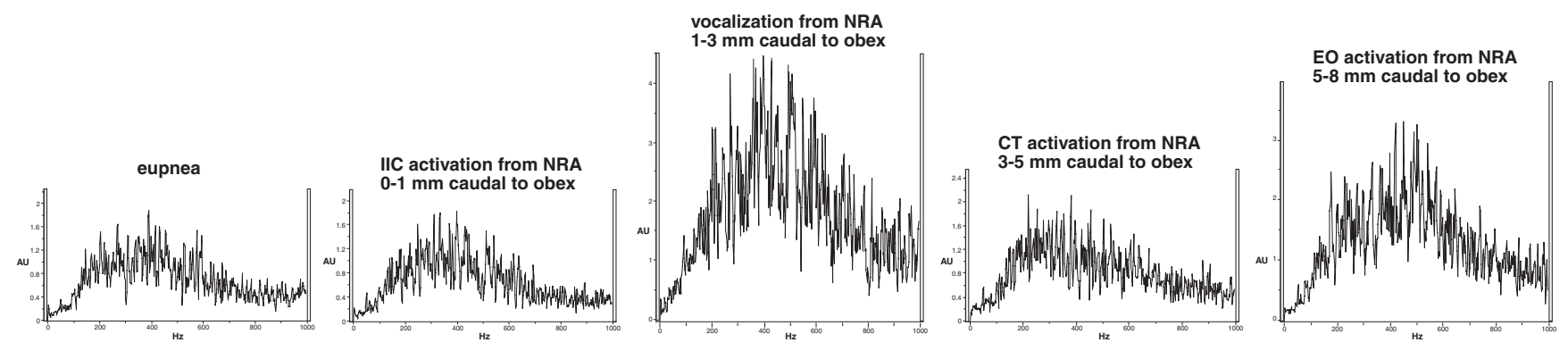

crural diaphragm EMG spectral analyses

Figure 9. Amplitude spectrum of the diaphragm EMG during eupneic inspiration and during NRA-induced respiratory effect. The $y$-axis is represented in arbitrary units (AU), and the $x$-axis shows the frequency spread of the EMG signal. The spectrum is represented as connected points in a Cosine-Bell data window. Note the more or less similar pattern of the amplitude spectrum during the effects compared with eupnea.

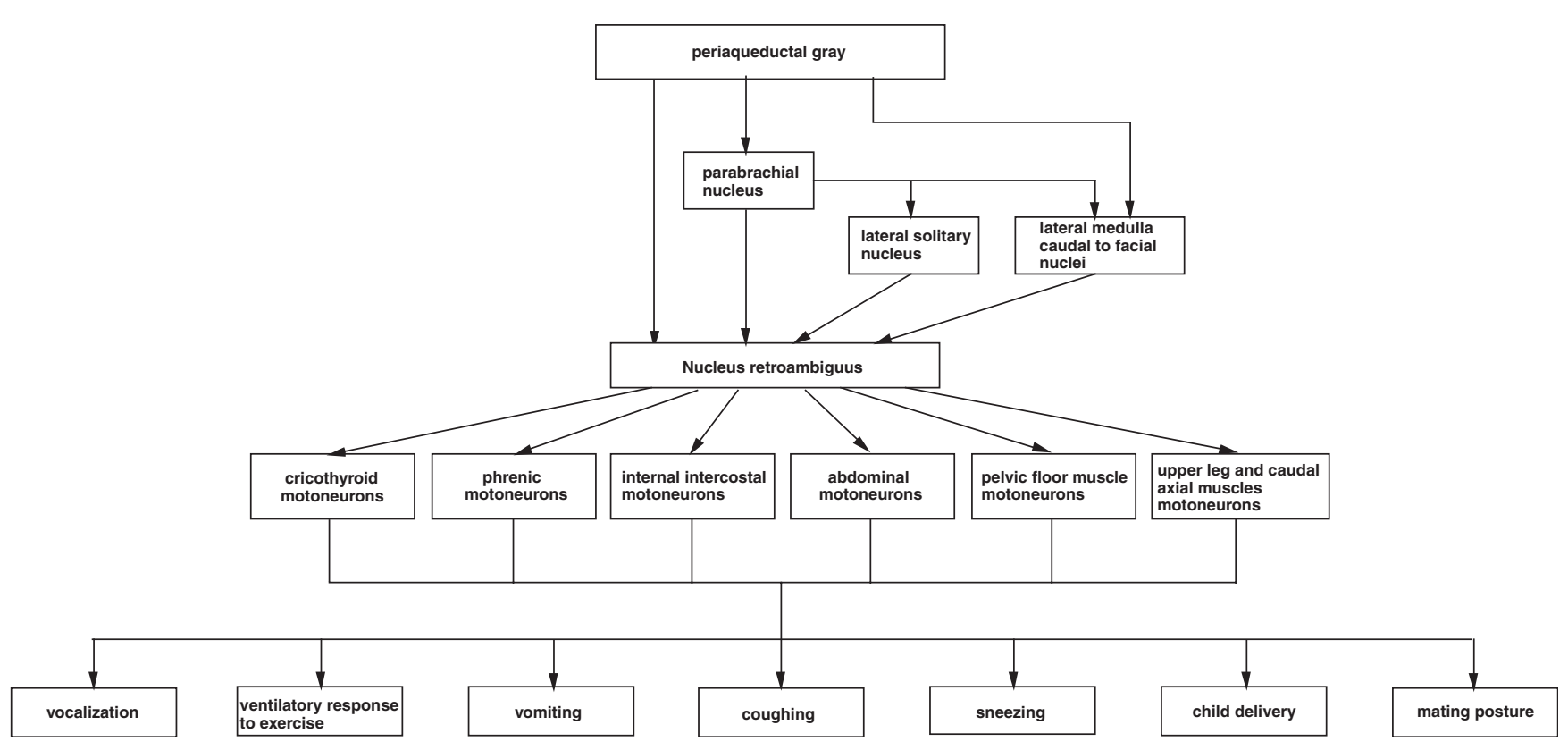

Figure 10. Diagram showing the motor functions mediated by the NRA.

panied by a significant increase in inspiratory duration, resulting in a decrease in respiratory rate. Possibly, the increase in inspiratory duration is brought about by NRA neurons activating the motoneurons of inspiration-related external intercostal muscles.

\section{Piano and piano player}

The present results clearly show that the NRA is not a central pattern generator. The NRA is nothing but a group of premotor interneurons with direct connections to motoneurons and stimulation of which produces components of different motor activities. The feature that NRA cells have in common is that the motor activities involve changes in abdominal pressure and thus need to change eupneic respiration into another breathing pattern. In none of the experiments, stimulation of the NRA neurons induced a coordinated motor activity. The necessary coordination, is, therefore, brought about by higher brainstem regions that have direct access to the NRA. These brainstem regions, such as the PAG, determine, by selectively exciting specific NRA neurons, what the final motor output of the NRA is. These higher brainstem structures decide whether to produce vocalization, vomiting, childbirth, coughing, sneezing or mating behavior, depending on what is necessary in that particular circumstance (Fig.
10). Actually, the NRA plays an important part in the lateral component of the emotional motor system (Holstege, 1992). Other pathways in this component are the projections of the PAG to the pontine micturition center that has direct access to the bladder motoneurons (Blok and Holstege, 1994), or the PAG projections to the retrofacial nucleus that has direct access to the sympathetic preganglionic motoneurons in the thoracic and upper lumbar cord that control blood pressure (Carrive et al. 1988). In simple terms, the NRA neurons together represent the piano, each neuron, by way of its motoneuronal projection, producing a very specific motor action, a tone. Higher regions in the brainstem (Gerrits and Holstege, 1996; Subramanian et al. 2008) play the role of the piano player. They, by means of their direct access to all parts of the NRA (piano), determine which cells (tones) will be excited (played) to produce the coordinated motor action (musical composition).

\section{References}

Berman AL (1968) The brainstem of the cat: a cytoarchitectonic atlas with stereotaxic coordinates. Madison, WI: University of Wisconsin.

Blok BF, Holstege G (1994) Direct projections from the periaqueductal gray to the pontine micturition center (M-region). An anterograde and retrograde tracing study in the cat. Neurosci Lett 166:93-96. 
Boers J, Klop EM, Hulshoff AC, de Weerd H, Holstege G (2002) Direct projections from the nucleus retroambiguus to cricothyroid motoneurons in the cat. Neurosci Lett 319:5-8.

Boers J, Kirkwood PA, de Weerd H, Holstege G (2006) Ultrastructural evidence for direct excitatory retroambiguus projections to cutaneous trunci and abdominal external oblique muscle motoneurons in the cat. Brain Res Bull 68:249-256.

Carrive P, Bandler R, Dampney RA (1988) Anatomical evidence that hypertension associated with the defence reaction in the cat is mediated by a direct projection from a restricted portion of the midbrain periaqueductal grey to the subretrofacial nucleus of the medulla. Brain Res 460:339-345.

de Troyer A, Kirkwood PA, Wilson TA (2005) Respiratory action of intercostal muscles. Physiol Rev 85:17-56.

Ford TW, Kirkwood PA (2006) Respiratory drive in hindlimb motoneurons of the anaesthetized female cat. Brain Res Bull 70:450-456.

Gerrits PO, Holstege G (1996) Pontine and medullary projections to the nucleus retroambiguus: a wheat germ agglutinin-horseradish peroxidase and autoradiographic tracing study in the cat. J Comp Neurol 373:173-185.

Holstege G (1989) Anatomical study of the final common pathway for vocalization in the cat. J Comp Neurol 284:242-252.

Holstege G (1991) Descending motor pathways and the spinal motor system. Limbic and non-limbic components. In: Progress in brain research, Vol 87, pp 307-421. Amsterdam: Elsevier.

Holstege G (1992) The emotional motor system. Eur J Morph 30:67-81.

Holstege G, Kuypers HG (1982) The anatomy of brainstem pathways to the spinal cord in cat. A labeled amino acid tracing study. Prog. Brain Res 57:145-175.

Holstege G, Tan J (1987) Supraspinal control of motoneurons innervating the striated muscles of the pelvic floor including urethral and anal sphincters in the cat. Brain 110:1323-1344.

Long S, Duffin J (1986) Neuronal determinants of respiratory rhythm. Prog Neurobiol 27:101-182.

Merril EG (1970) The lateral respiratory neurons of the medulla: their associations with NA, NRA and the spinal accessory nucleus and the spinal cord. Brain Res 24:11-28.
Merril EG (1974) Finding a respiratory function for the medullary respiratory neurons. In: Essays on the nervous system (Bellairs R, Gray EG, eds), pp 451-486. Oxford: Clarendon.

Miller AD, Ezure K, Suzuki I (1985) Control of abdominal muscles by brainstem respiratory neurons in the cat. J Neurophysiol 54:155-167.

Miller AD, Tan LK, Suzuki I (1987) Control of abdominal and expiratory intercostal muscle activity during vomiting: role of ventral respiratory group expiratory neurons. J Neurophysiol 57:1854-1866.

Miller AD, Nonaka S, Siniaia MS, Jakus J (1995) Multifunctional ventral respiratory group: bulbospinal expiratory neurons play a role in pudendal discharge during vomiting. J Auton Nerv Syst 54:253-260.

Olszewski J, Baxter D (1954) Cytoarchitecture of the human brain stem. Basel: Lippincott.

Rikard-Bell GC, Bystrzycka EK, Nail BS (1984) Brainstem projections to the phrenic nucleus: a HRP study in the cat. Brain Res Bull 12:469-477.

Rikard-Bell GC, Bystrzycka EK, Nail BS (1985) The identification of brainstem neurones projecting to thoracic respiratory motoneurons in the cat as demonstrated by retrograde transport of HRP. Brain Res Bull 14:25-37.

Saywell SA, Anissimova NP, Ford TW, Meehan CF, Kirkwood PA (2007) The respiratory drive to thoracic motoneurons in the cat and its relation to the connections from expiratory bulbospinal neurones. J Physiol 579:765-782.

Subramanian HH, Balnave RJ, Holstege G (2008) The midbrain periaqueductal gray control of respiration. J Neurosci 28:12274-12283.

Vanderhorst VG, Holstege G (1995) Caudal medullary pathways to lumbosacral motoneuronal cell groups in the cat: evidence for direct projections possibly representing the final common pathway for lordosis. J Comp Neurol 359:457-475.

Van der Horst VG, Holstege G (1998) Sensory and motor components of reproductive behavior: pathways and plasticity. Behav Brain Res 92:157-167.

Zhang SP, Bandler R, Davis PJ (1995) Brain stem integration of vocalization: Role of the nucleus retroambigualis. J Neurophysiol 74:2500-2512. 